\title{
A Multiple Iterated Integral Inequality and Applications
}

\author{
Zongyi Hou and Wu-Sheng Wang \\ School of Mathematics and Statistics, Hechi University, Guangxi, Yizhou 546300, China \\ Correspondence should be addressed to Wu-Sheng Wang; wang4896@126.com
}

Received 11 June 2014; Accepted 10 July 2014; Published 22 July 2014

Academic Editor: Daoyi Xu

Copyright (C) 2014 Z. Hou and W.-S. Wang. This is an open access article distributed under the Creative Commons Attribution License, which permits unrestricted use, distribution, and reproduction in any medium, provided the original work is properly cited.

We establish new multiple iterated Volterra-Fredholm type integral inequalities, where the composite function $w(u(s))$ of the unknown function $u$ with nonlinear function $w$ in integral functions in [Ma, QH, Pečarić, J: Estimates on solutions of some new nonlinear retarded Volterra-Fredholm type integral inequalities. Nonlinear Anal. 69 (2008) 393-407] is changed into the composite functions $w_{1}(u(s)), w_{2}(u(s)), \ldots, w_{n}(u(s))$ of the unknown function $u$ with different nonlinear functions $w_{1}, w_{2}, \ldots, w_{n}$, respectively. By adopting novel analysis techniques, the upper bounds of the embedded unknown functions are estimated explicitly. The derived results can be applied in the study of solutions of ordinary differential equations and integral equations.

\section{Introduction}

The well-known Gronwall-Bellman inequality $[1,2]$ is the following or can be equivalently regarded as the following:

$$
u(t) \leq c+\int_{a}^{t} f(s) u(s) d s, \quad t \in[a, a+T]
$$

where $c \geq 0$ is a constant, $f$ is a given nonnegative continuous function, and $u$ is the unknown function. It is often used to estimate solutions of differential equations. In 1956 Bihari [3] discussed

$$
u(t) \leq c+\int_{0}^{t} f(s) w(u(s)) d s
$$

In 1990 Pinto [4] investigated

$$
u(t) \leq c(t)+\sum_{i=1}^{n} \int_{a}^{t} g_{i}(s) w_{i}(u(s)) d s
$$

Replacing the upper limit $t$ of the integral with a function $b(t)$ in (2), in 2000 Lipovan [5] improved Bihari's results by investigating the following so-called retarded Gronwall-like inequalities:

$$
\begin{gathered}
u(t) \leq c+\int_{b\left(t_{0}\right)}^{b(t)} f(s) w(u(s)) d s, \\
u(t) \leq c+\int_{t_{0}}^{t} f(s) w(u(s)) d s+\int_{b\left(t_{0}\right)}^{b(t)} g(s) w(u(s)) d s .
\end{gathered}
$$

In 2005 Agarwal et al. [6] generally discussed

$$
u(t) \leq c(t)+\sum_{i=1}^{n} \int_{b_{i}\left(t_{0}\right)}^{b_{i}(t)} g_{i}(t, s) w_{i}(u(s)) d s, \quad t_{0} \leq t<t_{1} .
$$

As required in estimation for solutions, invariant sets, and stability, many generalized versions of the Gronwall-Bellman inequality were given with an invariant decomposition [7-9], a singular kernel $[10,11]$, and maxima $[12,13]$. More results about integral inequalities of single variable and multivariables can be found, for example, the books [14, 15].

In order to investigate the behavior of solutions of a linear Volterra-Fredholm type integral equation, a form of 
integral inequalities which contains multiple integrals of the unknown,

$u(t)$

$$
\begin{array}{r}
\leq c+\int_{\alpha\left(t_{0}\right)}^{\alpha(T)} b(t, s) u(s) d s \\
+\int_{\alpha\left(t_{0}\right)}^{\alpha(t)} a(t, s)\left[f(s) u(s)+\int_{\alpha\left(t_{0}\right)}^{s} c(s, \tau) u(\tau) d \tau\right] d s, \\
\forall t \in\left[t_{0}, T\right],
\end{array}
$$

called linear Volterra-Fredholm type integral inequality with retardation, is discussed by Pachpatte [16] in 2004.

In $2008 \mathrm{Ma}$ and Pečarić [17] discussed more generally the following inequality:

$$
\begin{aligned}
u(t) \leq c+ & \int_{\alpha\left(t_{0}\right)}^{\alpha(t)} \sigma_{1}(s) \\
& \times\left[f(s) w(u(s))+\int_{\alpha\left(t_{0}\right)}^{s} \sigma_{2}(\tau) w(u(\tau)) d \tau\right] d s \\
+ & \int_{\alpha\left(t_{0}\right)}^{\alpha(T)} \sigma_{1}(s) \\
& \times\left[f(s) w(u(s))+\int_{\alpha\left(t_{0}\right)}^{s} \sigma_{2}(\tau) w(u(\tau)) d \tau\right] d s, \\
& \quad \forall t \in I,
\end{aligned}
$$

where $I=\left[t_{0}, T\right]$. In 2011 Abdeldaim and Yakout [18] investigated the following:

$$
\begin{aligned}
& u(t) \\
& \leq c+\int_{\alpha\left(t_{0}\right)}^{t} f(s) u(s) \\
& \quad \times\left[u(s)+\int_{\alpha\left(t_{0}\right)}^{s} h(\tau)\left[u(\tau)+\int_{\alpha\left(t_{0}\right)}^{\tau} g(\xi) u(\xi) d \xi\right] d \tau\right] d s
\end{aligned}
$$

In 2013 Wang et al. [19] studied a new integral inequality of Gronwall-Bellman-Pachpatte type

$$
\begin{aligned}
\varphi(u(t)) \leq & c(t) \\
& +\int_{\alpha\left(t_{0}\right)}^{\alpha(t)} f_{1}(t, s) w_{1}(u(s)) d s \\
& +\int_{\alpha\left(t_{0}\right)}^{\alpha(t)} f_{1}(t, s) w_{1}(u(s)) \\
& \quad \times\left(\int_{t_{0}}^{s} f_{2}(s, \tau) w_{2}(u(\tau)) d \tau\right) d s
\end{aligned}
$$

$$
\begin{aligned}
& +\int_{\alpha\left(t_{0}\right)}^{\alpha(t)} f_{1}(t, s) w_{1}(u(s)) \\
& \quad \times\left(\int_{t_{0}}^{s} f_{2}(s, \tau) w_{2}(u(\tau))\right. \\
& \left.\quad \times\left(\int_{t_{0}}^{\tau} f_{3}(\tau, \xi) w_{3}(u(\xi)) d \xi\right) d \tau\right) d s
\end{aligned}
$$

In this paper, on the basis of $[17,18]$, we discuss a new multiple iterated Volterra-Fredholm type integral inequality

$$
\begin{aligned}
& u(t) \leq c+\int_{\alpha\left(t_{0}\right)}^{\alpha(t)} h_{1}\left(t_{1}\right) \\
& \times\left[f_{1}\left(t_{1}\right) \varphi_{1}\left(u\left(t_{1}\right)\right)+\int_{\alpha\left(t_{0}\right)}^{t_{1}} h_{2}\left(t_{2}\right)\right. \\
& \times\left[f_{2}\left(t_{2}\right) \varphi_{2}\left(u\left(t_{2}\right)\right)+\cdots\right. \\
& +\int_{\alpha\left(t_{0}\right)}^{t_{n-2}} h_{n-1}\left(t_{n-1}\right) \\
& \times\left[f_{n-1}\left(t_{n-1}\right) \varphi_{n-1}\left(u\left(t_{n-1}\right)\right)\right. \\
& \left.+\int_{\alpha\left(t_{0}\right)}^{t_{n-1}} h_{n}\left(t_{n}\right) \varphi_{n}\left(u\left(t_{n}\right)\right) d t_{n}\right] \\
& \left.\left.\times d t_{n-1} \cdots\right] d t_{2}\right] d t_{1} \\
& +\int_{\alpha\left(t_{0}\right)}^{\alpha(T)} h_{1}\left(t_{1}\right)\left[f_{1}\left(t_{1}\right) \varphi_{1}\left(u\left(t_{1}\right)\right)+\int_{\alpha\left(t_{0}\right)}^{t_{1}} h_{2}\left(t_{2}\right)\right. \\
& \times\left[f_{2}\left(t_{2}\right) \varphi_{2}\left(u\left(t_{2}\right)\right)+\cdots\right. \\
& +\int_{\alpha\left(t_{0}\right)}^{t_{n-2}} h_{n-1}\left(t_{n-1}\right) \\
& \times\left[f_{n-1}\left(t_{n-1}\right) \varphi_{n-1}\left(u\left(t_{n-1}\right)\right)\right. \\
& +\int_{\alpha\left(t_{0}\right)}^{t_{n-1}} h_{n}\left(t_{n}\right) \varphi_{n}\left(u\left(t_{n}\right)\right) \\
& \left.\left.\left.\times d t_{n}\right] d t_{n-1} \cdots\right] d t_{2}\right] d t_{1} .
\end{aligned}
$$

Using monotonization of some functions, we simplify the above multicomposition in an operator form. The unknown function will be estimated by known functions. Furthermore, we apply our result to retarded nonlinear Volterra-Fredholm type equations for estimation of solutions. 


\section{Preliminaries}

Throughout this paper, let $\mathbb{R}$ denote the set of real numbers, $\mathbb{R}_{+}=[0,+\infty)$ and $I=\left[t_{0}, T\right]$. For $k=0,1$, let $C^{k}(M, S)$ denote the class of $k$ th order continuously differentiable functions defined on the set $M$ and ranged in the set $S$. For simplicity, we use the product $\prod_{i=1}^{n} f_{i}$ and $\prod_{i=n}^{1} f_{i}$ to present the composition $f_{1} \circ f_{2} \circ \cdots \circ f_{n}$ and $f_{n} \circ f_{n-1} \circ \cdots \circ f_{1}$.

2.1. Monotonization. First, we monotonize those $\varphi_{i}$ s in inequality (11). Define

$$
\begin{aligned}
w_{1}(u) & :=\max _{\tau \in[0, u]}\left\{\varphi_{1}(\tau)\right\}, \\
w_{i+1}(u) & :=\max _{\tau \in[0, u]}\left\{\frac{\varphi_{i+1}(\tau)}{w_{i}(\tau)}\right\} w_{i}(u), \quad i=1, \ldots, n,
\end{aligned}
$$

recursively. One can prove that

(P1) each $w_{i}$ is a nondecreasing nonnegative continuous function,

(P2) $\varphi_{i}(u) \leq w_{i}(u), i=1, \ldots, n$,

(P3) $w_{i+1}$ has stronger monotonicity than $w_{i}$, denoted by $w_{i} \propto w_{i+1}, i=1,2, \ldots, n-1$; that is, by the definition given in $[4,6]$, the ratios $w_{i+1}(u) / w_{i}(u), i=1, \ldots, n-1$, are all nondecreasing.

Thus, the sequence $\left\{\varphi_{i}\right\}$ can be replaced by a larger but monotonous one $\left\{w_{i}\right\}$ in (11). For a given constant $u_{i}>0$, define functions $W_{i}\left(u, u_{1}\right), i=1,2, \ldots, n$, recursively by

$$
\begin{aligned}
W_{1}\left(u, u_{1}\right) & :=\int_{u_{1}}^{u} \frac{d s}{w_{1}(s)}, \\
W_{i}\left(u, u_{i}\right) & :=\int_{u_{i}}^{u} \frac{w_{i-1}\left(\prod_{\ell=1}^{i-1} W_{\ell}^{-1}(s)\right) d s}{w_{i}\left(\prod_{\ell=1}^{i-1} W_{\ell}^{-1}(s)\right)}, \quad i=2, \ldots, n,
\end{aligned}
$$

where we use $W_{\ell}$ and $W_{\ell}^{-1}$ to denote $W_{\ell}\left(u, u_{\ell}\right)$ and its inverse when there is no confusion. Clearly, they are all strictly increasing.

For given positive constants $c_{i}, i=1,2, \ldots, n$, define $\widehat{\mathscr{W}}_{i}(u)$ by

$$
\widehat{\mathscr{W}}_{i}(u):=W_{i}(u)+c_{i}, \quad \forall u \in \mathbb{R}_{+}, i=1,2, \ldots, n,
$$

where $W_{i}(i=1,2, \ldots, n)$ are defined by (13) and (14), respectively.
Lemma 1. Suppose that $\varphi_{i}(i=1,2, \ldots, n)$ are nonnegative and integrable on $\left[t_{0}, T\right]$. Then $W_{i}(i=1,2, \ldots, n)$ are increasing and continuous differentiable functions, and

$$
\begin{gathered}
\frac{d}{d \xi}\left(\prod_{i=n}^{2} W_{i} \circ W_{1}(\xi)\right)=\frac{1}{w_{n}(\xi)}, \\
\frac{d}{d \xi}\left(\prod_{i=n}^{2} \widehat{\mathscr{W}}_{i} \circ \widehat{\mathscr{W}}_{1}(\xi)\right) \\
=\frac{w_{n-1}\left(\prod_{i=1}^{n-1} W_{i}^{-1} \circ\left(\prod_{i=n-1}^{2} \widehat{\mathscr{W}}_{i} \circ \widehat{\mathscr{W}}_{1}(\xi)\right)\right)}{w_{n}\left(\prod_{i=1}^{n-1} W_{i}^{-1} \circ\left(\prod_{i=n-1}^{2} \widehat{\mathscr{W}}_{i} \circ \widehat{\mathscr{W}}_{1}(\xi)\right)\right)} \\
\ldots \frac{w_{1}\left(W_{1}^{-1} \circ \widehat{\mathscr{W}}_{1}(\xi)\right)}{w_{2}\left(W_{1}^{-1} \circ \widehat{\mathscr{W}}_{1}(\xi)\right)} \frac{1}{w_{1}(\xi)}, \quad \forall \xi \geq k(T) .
\end{gathered}
$$

Proof. By the definition, $W_{i}(s)(i=1,2, \ldots, n)$ are increasing and continuous differentiable functions. From (13) and (14), we have

$$
\begin{aligned}
\frac{d}{d \xi}( & \left.\prod_{i=n}^{2} W_{i} \circ W_{1}(\xi)\right) \\
= & W_{n}^{\prime}\left(\prod_{i=n-1}^{1} W_{i}(\xi) \cdots\right) \cdots W_{2}^{\prime}\left(W_{1}(\xi)\right) W_{1}^{\prime}(\xi) \\
= & \frac{w_{n-1}\left(\prod_{i=1}^{n-1} W_{i}^{-1}\left(\prod_{i=n-1}^{1} W_{i}(\xi)\right)\right)}{w_{n}\left(\prod_{i=1}^{n-1}\left(W_{i}^{-1}\left(\prod_{i=n-1}^{1} W_{i}(\xi)\right)\right)\right)} \\
& \ldots \frac{w_{1}\left(W_{1}^{-1}\left(W_{1}(\xi)\right)\right)}{w_{2}\left(W_{1}^{-1}\left(W_{1}(\xi)\right)\right)} \frac{1}{w_{1}(\xi)} \\
= & \frac{1}{w_{n}(\xi)}
\end{aligned}
$$

Moreover,

$$
\begin{aligned}
& \frac{d}{d \xi}\left(\prod_{i=n}^{2} \widehat{\mathscr{W}}_{i} \circ \widehat{\mathscr{W}}_{1}(\xi)\right) \\
& =\frac{d\left(W_{n}\left(W_{n-1}\left(\cdots\left(W_{2}\left(W_{1}(\xi)+c_{1}\right)+c_{2}\right) \cdots\right)+c_{n-1}\right)+c_{n}\right)}{d \xi} \\
& =W_{n}^{\prime}\left(W_{n-1}\left(\cdots\left(W_{2}\left(W_{1}(\xi)+c_{1}\right)+c_{2}\right) \cdots\right)+c_{n-1}\right) \\
& \quad \cdots W_{2}^{\prime}\left(W_{1}(\xi)+c_{1}\right) W_{1}^{\prime}(\xi) \\
& =w_{n-1}\left(\prod _ { i = 1 } ^ { n - 1 } W _ { i } ^ { - 1 } \left(W _ { n - 1 } \left(\cdots \left(W_{2}\left(W_{1}(\xi)+c_{1}\right)\right.\right.\right.\right. \\
& \left.\left.\left.\left.\quad+c_{2}\right) \cdots\right)+c_{n-1}\right)\right)
\end{aligned}
$$




$$
\begin{aligned}
& \times\left(w _ { n } \left(\prod _ { i = 1 } ^ { n - 1 } W _ { i } ^ { - 1 } \left(W_{n-1}\left(\cdots\left(W_{2}\left(W_{1}(\xi)+c_{1}\right)+c_{2}\right) \cdots\right)\right.\right.\right. \\
& \left.\left.\left.+c_{n-1}\right)\right)\right)^{-1} \\
& \cdots \frac{w_{1}\left(W_{1}^{-1}\left(W_{1}(\xi)+c_{1}\right)\right)}{w_{2}\left(W_{1}^{-1}\left(W_{1}(\xi)+c_{1}\right)\right)} \frac{1}{w_{1}(\xi)} .
\end{aligned}
$$

By the definitions of $\widehat{\mathscr{W}}_{i}(u)$, we have the relation (17).

2.2. Simplification with Operators. Let $h_{i}, f_{i}$ be positive continuous functions in (11), where $i=1,2, \ldots, n, f_{n}(t) \equiv 1$. Define $\mathscr{H}_{i}: C\left(\left[t_{0}, T\right]\right) \rightarrow C\left(\left[t_{0}, T\right]\right)$ by

$$
\mathscr{H}_{i} v(t):=\int_{\alpha\left(t_{0}\right)}^{t} h_{i}(s) v(s) d s, \quad i=1,2, \ldots, n,
$$

and define $\mathscr{F}_{i}[u]: C\left(\left[t_{0}, T\right]\right) \rightarrow C\left(\left[t_{0}, T\right]\right)$ by

$$
\mathscr{F}_{i}[u] v(t):=f_{i}(t) w_{i}(u(t))+v(t), \quad i=1,2, \ldots, n .
$$

Having defined those operators, we can enlarge inequality (11) by (12) in the simpler form

$$
\begin{array}{r}
u(t) \leq k(t) \\
+\left\{\prod_{i=1}^{n-1}\left(\mathscr{H}_{i} \circ \mathscr{F}_{i}[u]\right) \circ \mathscr{H}_{n} \circ \mathscr{F}_{n}[u] \mathbf{0}\right\}(\alpha(t)) \\
+\left\{\prod_{i=1}^{n-1}\left(\mathscr{H}_{i} \circ \mathscr{F}_{i}[u]\right) \circ \mathscr{H}_{n} \circ \mathscr{F}_{n}[u] \mathbf{0}\right\}(\alpha(T)), \\
\forall t \in\left[t_{0}, T\right],
\end{array}
$$

where $\mathbf{0}$ denotes a zero function.

2.3. $\mathscr{W}$-Function. Define $\mathscr{W}_{i}(u)(\alpha(T))$ by

$$
\begin{array}{r}
\mathscr{W}_{i}(u)(\alpha(T)):=W_{i}(u)+\left(\prod_{j=1}^{i-1} \mathscr{H}_{j}\right) \circ\left(\mathscr{H}_{i} f_{i}(t)\right)(\alpha(T)), \\
\forall u \in \mathbb{R}_{+}, \quad i=1,2, \ldots, n,
\end{array}
$$

where $W_{i}$ and $\mathscr{H}_{i}$ are defined by (14) and (20), respectively. Since $W_{i}(i=1,2, \ldots, n)$ are continuous functions, $\mathscr{W}_{i}(i=$ $1,2, \ldots, n)$ are also continuous functions.

Define a function

$$
\begin{aligned}
\widetilde{\mathscr{W}}(\xi):= & \prod_{i=n}^{2} W_{i} \circ W_{1}(2 \xi-c) \\
& -\prod_{i=n}^{2} \mathscr{W}_{i} \circ \mathscr{W}_{1}(\xi)(\alpha(T)), \quad \forall \xi>c .
\end{aligned}
$$

Lemma 2. Suppose that $\varphi_{i}(u)(i=1, \ldots, n)$ are all continuous such that $\varphi_{i}(u)>0$ for $u>0, W_{i}(i=1,2, \ldots, n)$ defined by
(13) and (14) satisfy $W_{i}(+\infty)=+\infty$. Suppose that $h_{i}, f_{i}$, and $\varphi_{i}(i=1, \ldots, n)$ satisfy

$$
\frac{1}{w_{n}(2 \xi-c)}-V(\xi) \geq 0, \quad \forall \xi>c,
$$

where

$$
V(\xi):
$$

$$
\begin{aligned}
= & \frac{w_{n-1}\left(\prod_{i=1}^{n-1} W_{i}^{-1} \circ\left(\prod_{i=n-1}^{2} \mathscr{W}_{i} \circ \mathscr{W}_{1}(\xi)(\alpha(T))\right)\right)}{w_{n}\left(\prod_{i=1}^{n-1} W_{i}^{-1} \circ\left(\prod_{i=n-1}^{2} \widetilde{\mathscr{W}}_{i} \circ \mathscr{W}_{1}(\xi)(\alpha(T))\right)\right)} \\
& \ldots \frac{w_{1}\left(W_{1}^{-1} \circ \mathscr{W}_{1}(\xi)(\alpha(T))\right)}{w_{2}\left(W_{1}^{-1} \circ \mathscr{W}_{1}(\xi)(\alpha(T))\right)} \frac{1}{w_{1}(\xi)},
\end{aligned}
$$

and $\mathscr{W}_{i}(i=1,2, \ldots, n)$ are defined by $(23)$. Then, $\widetilde{\mathscr{W}}(\xi)$ is nondecreasing, and $\widetilde{\mathscr{W}}(\xi)=0$ has a solution $\xi=\xi_{0}$ with $\xi_{0}>c$.

Proof. Using Lemma 1 and condition (25), we have

$$
\mathscr{W}^{\prime}(\xi)=\frac{1}{w_{n}(2 \xi-c)}-V(\xi) \geq 0 .
$$

Thus $\widetilde{\mathscr{W}}(\xi)$ is a nondecreasing function. Since $\widetilde{\mathscr{W}}(c)<0$ and $\lim _{\xi \rightarrow \infty} \widetilde{\mathscr{W}}(\xi)=+\infty$, we see that $\widetilde{\mathscr{W}}(\xi)=0$ has a solution $\xi=\xi_{0}$ with $\xi_{0}>c$.

\section{Main Result}

The following theorem shows that the unknown function is estimated by the given known functions.

Theorem 3. Let $c$ be a positive constant. Suppose that $h_{n}(t)$, $f_{i}(t)$, and $h_{i}(t) \in C\left(I, \mathbb{R}_{+}\right), i=1, \ldots, n-1$. Suppose that $h_{i}$, $f_{i}, h_{i}$, and $\varphi_{i}(i=1, \ldots, n)$ satisfy the assumption of Lemma 2 . Suppose that $\alpha \in C^{1}(I, I)$ is nondecreasing such that $\alpha(t) \leq t$ on $I$. Then the unknown $u(t)$ in (22) is estimated as

$$
u(t) \leq \prod_{i=1}^{n} W_{i}^{-1}\left\{\prod_{i=n}^{2} \mathscr{W}_{i} \circ \mathscr{W}_{1}\left(\xi_{0}\right)(\alpha(t))\right\}, \quad \forall t \in\left[t_{0}, T\right] .
$$

Remark 4. As explained in Remark 2 in [6], different choices of $u_{i}$ in the definitions (13)-(14) of $W_{i}$ do not affect our results (28).

Proof. For convenience, we cite some definitions in the discussion of our proof as follows: for each fixed positive continuous function $u$, define $\mathscr{F}_{i j}[u]: C\left(\left[t_{0}, T\right]\right) \rightarrow$ $C\left(\left[t_{0}, T\right]\right)$ by

$$
\begin{aligned}
& \mathscr{F}_{i j}[u] v(t): \\
& =f_{i}(t) \frac{w_{i}\left(W_{1}^{-1}\left(\cdots\left(W_{j-1}^{-1}(u(t))\right) \cdots\right)\right)}{w_{j}\left(W_{1}^{-1}\left(\cdots\left(W_{j-1}^{-1}(u(t))\right) \cdots\right)\right)}+v(t),
\end{aligned}
$$

where $j<i, i=2, \ldots, n, j=1,2, \ldots, n-1$. 
From (22), we have

$$
\begin{array}{r}
u(t) \\
\leq c+\left\{\left(\prod_{i=1}^{n-1} \mathscr{H}_{i} \circ \mathscr{F}_{i}[u]\right) \circ \mathscr{H}_{n} \circ \mathscr{F}_{n}[u] \mathbf{0}\right\}(\alpha(t)) \\
+\left\{\left(\prod_{i=1}^{n-1} \mathscr{H}_{i} \circ \mathscr{F}_{i}[u]\right) \circ \mathscr{H}_{n} \circ \mathscr{F}_{n}[u] \mathbf{0}\right\}(\alpha(T)), \\
\forall t \in\left[t_{0}, T_{1}\right] .
\end{array}
$$

Define a function $z_{1}(t)$ by the function on the right-hand side of (30). Then, $z_{1}(t)$ is a positive and nondecreasing function on $\left[t_{0}, T\right]$. Using $(30)$, we have

$$
\begin{aligned}
u(t) & \leq z_{1}(t), \quad \forall t \in\left[t_{0}, T\right], \\
z_{1}\left(t_{0}\right) & =c+\left\{\left(\prod_{i=1}^{n-1} \mathscr{H}_{i} \circ \mathscr{F}_{i}[u]\right) \circ \mathscr{H}_{n} \circ \mathscr{F}_{n}[u] \mathbf{0}\right\}(\alpha(T)) .
\end{aligned}
$$

Differentiating $z_{1}(t)$ with respect to $t$, using (31) we have

$$
\begin{aligned}
z_{1}^{\prime}(t)= & \alpha^{\prime}(t) h_{1}(\alpha(t)) \\
\times & {\left[f_{1}(\alpha(t)) w_{1}(u(\alpha(t)))\right.} \\
& \left.+\left[\left(\prod_{i=2}^{n-1} \mathscr{H}_{i} \circ \mathscr{F}_{i}[u]\right) \circ \mathscr{H}_{n} \circ \mathscr{F}_{n}[u] \mathbf{0}\right](\alpha(t))\right] \\
\leq & \alpha^{\prime}(t) h_{1}(\alpha(t)) \\
\times & {\left[f_{1}(\alpha(t)) w_{1}\left(z_{1}(\alpha(t))\right)\right.} \\
& \left.+\left[\left(\prod_{i=2}^{n-1} \mathscr{H}_{i} \circ \mathscr{F}_{i}\left[z_{1}\right]\right) \circ \mathscr{H}_{n} \circ \mathscr{F}_{n}\left[z_{1}\right] \mathbf{0}\right](\alpha(t))\right],
\end{aligned}
$$

for all $t \in\left[t_{0}, T\right]$. From (33), we have

$$
\begin{aligned}
& \frac{z_{1}^{\prime}(t)}{w_{1}\left(z_{1}(t)\right)} \leq \alpha^{\prime}(t) h_{1}(\alpha(t)) \\
& \times\left[f_{1}(\alpha(t))+\left[\left(\prod_{i=2}^{n-1} \mathscr{H}_{i} \circ \mathscr{F}_{i 1}\left[z_{1}\right]\right)\right.\right. \\
& \\
&\left.\left.\quad \circ \mathscr{H}_{n} \circ \mathscr{F}_{n 1}\left[z_{1}\right] \mathbf{0}\right](\alpha(t))\right],
\end{aligned}
$$

for all $t \in\left[t_{0}, T\right]$. Integrating both sides of the above inequality from $t_{0}$ to $t$, we have

$$
\begin{aligned}
W_{1} & \left(z_{1}(t)\right) \\
\leq & W_{1}\left(z_{1}\left(t_{0}\right)\right)+\mathscr{H}_{1}\left(f_{1}(s)\right)(\alpha(t)) \\
& +\left[\mathscr{H}_{1} \circ\left(\prod_{i=2}^{n-1} \mathscr{H}_{i} \circ \mathscr{F}_{i 1}\left[z_{1}\right]\right) \circ \mathscr{H}_{n} \circ \mathscr{F}_{n 1}\left[z_{1}\right] \mathbf{0}\right](\alpha(t)) \\
\leq & W_{1}\left(z_{1}\left(t_{0}\right)\right)+\mathscr{H}_{1}\left(f_{1}(s)\right)(\alpha(T)) \\
& +\left[\mathscr{H}_{1} \circ\left(\prod_{i=2}^{n-1} \mathscr{H}_{i} \circ \mathscr{F}_{i 1}\left[z_{1}\right]\right) \circ \mathscr{H}_{n} \circ \mathscr{F}_{n 1}\left[z_{1}\right] \mathbf{0}\right](\alpha(t)),
\end{aligned}
$$

for $t \in\left[t_{0}, T\right]$.

Let $z_{2}(t)$ denote the function on the right-hand side of (35); we can see that $z_{2}(t)$ is a positive and nondecreasing function on $\left[t_{0}, T\right]$. From (35), we obtain

$$
\begin{gathered}
z_{1}(t) \leq W_{1}^{-1}\left(z_{2}(t)\right), \quad \forall t \in\left[t_{0}, T\right], \\
z_{2}\left(t_{0}\right)=W_{1}\left(z_{1}\left(t_{0}\right)\right)+\left(\mathscr{H}_{1} f_{1}(s)\right)(\alpha(T)) .
\end{gathered}
$$

Differentiating $z_{2}(t)$ with respect to $t$, using (36) we obtain

$$
\begin{aligned}
& z_{2}^{\prime}(t) \\
& =\alpha^{\prime}(t) h_{1}(\alpha(t)) \\
& \times\left[\int _ { \alpha ( t _ { 0 } ) } ^ { \alpha ( t ) } h _ { 2 } ( t _ { 2 } ) \left[f_{2}\left(t_{2}\right) \frac{w_{2}\left(z_{1}\left(t_{2}\right)\right)}{w_{1}\left(z_{1}\left(t_{2}\right)\right)}\right.\right. \\
& +\left[\left(\prod_{i=3}^{n-1} \mathscr{H}_{i} \circ \mathscr{F}_{i 1}\left[z_{1}\right]\right)\right. \\
& \left.\left.\left.\circ \mathscr{H}_{n} \circ \mathscr{F}_{n 1}\left[z_{1}\right] \mathbf{0}\right]\right] d t_{2}\right] \\
& \leq \alpha^{\prime}(t) h_{1}(\alpha(t))\left[\int_{\alpha\left(t_{0}\right)}^{\alpha(t)} h_{2}\left(t_{2}\right)\right. \\
& \times\left[f_{2}\left(t_{2}\right) \frac{w_{2}\left(W_{1}^{-1}\left(z_{2}\left(t_{2}\right)\right)\right)}{w_{1}\left(W_{1}^{-1}\left(z_{2}\left(t_{2}\right)\right)\right)}\right. \\
& +\left[\left(\prod_{i=3}^{n-1} \mathscr{H}_{i} \circ \mathscr{F}_{i 1}\left[W_{1}^{-1} \circ z_{2}\right]\right) \circ \mathscr{H}_{n}\right. \\
& \left.\left.\left.\circ \mathscr{F}_{n 1}\left[W_{1}^{-1} \circ z_{2}\right] \mathbf{0}\right]\right] d t_{2}\right] \text {, } \\
& \forall t \in\left[t_{0}, T\right] \text {. }
\end{aligned}
$$


From (38), we have

$$
\begin{aligned}
& \frac{z_{2}^{\prime}(t) w_{1}\left(W_{1}^{-1}\left(z_{2}(t)\right)\right)}{w_{2}\left(W_{1}^{-1}\left(z_{2}(t)\right)\right)} \\
& \leq \alpha^{\prime}(t) h_{1}(\alpha(t)) \int_{\alpha\left(t_{0}\right)}^{\alpha(t)} h_{2}\left(t_{2}\right) f_{2}\left(t_{2}\right) d t_{2} \\
& +\alpha^{\prime}(t) h_{1}(\alpha(t))\left[\mathscr{H}_{2} \circ\left(\prod_{i=3}^{n-1} \mathscr{H}_{i} \circ \mathscr{F}_{i 2}\left[z_{2}\right]\right)\right. \\
& \left.{ }^{\circ} \mathscr{H}_{n} \circ \mathscr{F}_{n 2}\left[z_{2}\right] \mathbf{0}\right](\alpha(t)),
\end{aligned}
$$

for all $t \in\left[t_{0}, T\right]$. From (39), we have

$$
\begin{aligned}
W_{2}\left(z_{2}(t)\right) \leq W_{2}\left(z_{2}\left(t_{0}\right)\right) \\
+\left[\mathscr{H}_{1} \circ\left(\mathscr{H}_{2} f_{2}(s)\right)\right](\alpha(t)) \\
+\left[\mathscr{H}_{1} \circ \mathscr{H}_{2} \circ\left(\prod_{i=3}^{n-1} \mathscr{H}_{i} \circ \mathscr{F}_{i 2}\left[z_{2}\right]\right)\right. \\
\left.\circ \mathscr{H}_{n} \circ \mathscr{F}_{n 2}\left[z_{2}\right] \mathbf{0}\right](\alpha(t)) \\
\leq W_{2}\left(z_{2}\left(t_{0}\right)\right)+\left[\mathscr{H}_{1} \circ\left(\mathscr{H}_{2} f_{2}(s)\right)\right](\alpha(T)) \\
+\left[\mathscr{H}_{1} \circ \mathscr{H}_{2} \circ\left(\prod_{i=3}^{n-1} \mathscr{H}_{i} \circ \mathscr{F}_{i 2}\left[z_{2}\right]\right)\right. \\
\left.\circ \mathscr{H}_{n} \circ \mathscr{F}_{n 2}\left[z_{2}\right] \mathbf{0}\right](\alpha(t)),
\end{aligned}
$$

for all $t \in\left[t_{0}, T\right]$. Proceeding with the same derivation as in (36) to (40) and so on, we obtain

$$
\begin{aligned}
W_{n-2}\left(z_{n-2}(t)\right) \leq & W_{n-2}\left(z_{n-2}\left(t_{0}\right)\right) \\
+ & {\left[\left(\prod_{i=1}^{n-2} \mathscr{H}_{i}\right) f_{n-2}(s)\right](\alpha(t)) } \\
+ & {\left[\left(\prod_{i=1}^{n-2} \mathscr{H}_{i}\right) \circ \mathscr{H}_{n-1} \circ \mathscr{F}_{(n-1)(n-2)}\left[z_{n-2}\right]\right.} \\
& \left.\circ \mathscr{H}_{n} \circ \mathscr{F}_{n(n-2)}\left[z_{n-2}\right] \mathbf{0}\right](\alpha(t)) \\
\leq & W_{n-2}\left(z_{n-2}\left(t_{0}\right)\right) \\
& +\left[\left(\prod_{i=1}^{n-2} \mathscr{H}_{i}\right) f_{n-2}(s)\right](\alpha(T))
\end{aligned}
$$

$$
\begin{gathered}
+\left[\left(\prod_{i=1}^{n-2} \mathscr{H}_{i}\right) \circ \mathscr{H}_{n-1} \circ \mathscr{F}_{(n-1)(n-2)}\left[z_{n-2}\right]\right. \\
\left.\quad \circ \mathscr{H}_{n} \circ \mathscr{F}_{n(n-2)}\left[z_{n-2}\right] \mathbf{0}\right](\alpha(t)),
\end{gathered}
$$

for all $t \in\left[t_{0}, T\right]$, where $W_{n-2}$ is defined by (14).

Define a function $z_{n-1}(t)$ by the function on the righthand side of (41). Then $z_{n-1}(t)$ is a positive and nondecreasing function on $\left[t_{0}, T\right]$. From (41), we get

$$
z_{n-2}(t) \leq W_{n-2}^{-1}\left(z_{n-1}(t)\right), \quad \forall t \in\left[t_{0}, T\right],
$$

$$
\begin{aligned}
& z_{n-1}\left(t_{0}\right) \\
& \quad=W_{n-2}\left(z_{n-2}\left(t_{0}\right)\right)+\left[\left(\prod_{i=1}^{n-2} \mathscr{H}_{i}\right) f_{n-2}(s)\right](\alpha(T)) .
\end{aligned}
$$

Differentiating $z_{n-1}(t)$ with respect to $t$, we have

$$
\begin{aligned}
z_{n-1}^{\prime}(t)= & \alpha^{\prime}(t) h_{1}(\alpha(t)) \\
\times & {\left[\left(\prod_{i=2}^{n-1} \mathscr{H}_{i}\right) \circ \mathscr{F}_{(n-1)(n-2)}\left[z_{n-2}\right]\right.} \\
& \left.\circ \mathscr{H}_{n} \circ \mathscr{F}_{n(n-2)}\left[z_{n-2}\right] \mathbf{0}\right](\alpha(t)) \\
\leq & \alpha^{\prime}(t) h_{1}(\alpha(t)) \\
\times & {\left[\left(\prod_{i=2}^{n-1} \mathscr{H}_{i}\right) \circ \mathscr{F}_{(n-1)(n-2)}\left[W_{n-2}^{-1} \circ z_{n-1}\right]\right.} \\
& \left.\circ \mathscr{H}_{n} \circ \mathscr{F}_{n(n-2)}\left[W_{n-2}^{-1} \circ z_{n-1}\right] \mathbf{0}\right](\alpha(t)),
\end{aligned}
$$

for all $t \in\left[t_{0}, T\right]$. Then (44) is equivalent to

$$
\begin{aligned}
& \frac{z_{n-1}^{\prime}(t) w_{n-2}\left(W_{1}^{-1}\left(W_{2}^{-1}\left(\cdots\left(W_{n-2}^{-1}\left(z_{n-1}\left(t_{n-1}\right)\right)\right) \cdots\right)\right)\right)}{w_{n-1}\left(W_{1}^{-1}\left(W_{2}^{-1}\left(\cdots\left(W_{n-2}^{-1}\left(z_{n-1}\left(t_{n-1}\right)\right)\right) \cdots\right)\right)\right)} \\
& \leq \alpha^{\prime}(t) h_{1}(\alpha(t))\left[\left(\prod_{i=2}^{n-1} \mathscr{H}_{i}\right) f_{n-1}(s)\right](\alpha(t)) \\
& \quad+\alpha^{\prime}(t) h_{1}(\alpha(t)) \\
& \quad \times\left[\left(\prod_{i=2}^{n-1} \mathscr{H}_{i}\right) \circ \mathscr{H}_{n} \circ \mathscr{F}_{n(n-1)}\left[z_{n-1}\right] \mathbf{0}\right](\alpha(t)),
\end{aligned}
$$


for all $t \in\left[t_{0}, T\right]$. Integrating both sides of (45) from $t_{0}$ to $t$, we have

$$
\begin{aligned}
& W_{n-1}\left(z_{n-1}(t)\right) \\
& \leq W_{n-1}\left(z_{n-1}\left(t_{0}\right)\right)+\left[\left(\prod_{i=1}^{n-1} \mathscr{H}_{i}\right) f_{n-1}(s)\right](\alpha(t)) \\
& +\left[\left(\prod_{i=1}^{n-1} \mathscr{H}_{i}\right) \circ \mathscr{H}_{n} \circ \mathscr{F}_{n(n-1)}\left[z_{n-1}\right] \mathbf{0}\right](\alpha(t)) \\
& \leq W_{n-1}\left(z_{n-1}\left(t_{0}\right)\right)+\left[\left(\prod_{i=1}^{n-1} \mathscr{H}_{i}\right) f_{n-1}(s)\right](\alpha(T)) \\
& +\left[\left(\prod_{i=1}^{n-1} \mathscr{H}_{i}\right) \circ \mathscr{H}_{n} \circ \mathscr{F}_{n(n-1)}\left[z_{n-1}\right] \mathbf{0}\right](\alpha(t)),
\end{aligned}
$$

for all $t \in\left[t_{0}, T\right]$.

Define a function $z_{n}(t)$ by the function on the righthand side of (46); then, $z_{n}(t)$ is a positive and nondecreasing function on $\left[t_{0}, T\right]$. From (46), we have

$$
\begin{aligned}
& z_{n-1}(t) \leq W_{n-1}^{-1}\left(z_{n}(t)\right), \quad \forall t \in\left[t_{0}, T\right], \\
& z_{n}\left(t_{0}\right)=W_{n-1}\left(z_{n-1}\left(t_{0}\right)\right)+\left[\left(\prod_{i=1}^{n-1} \mathscr{H}_{i}\right) f_{n-1}(s)\right](\alpha(T)) .
\end{aligned}
$$

Differentiating $z_{n}(t)$ with respect to $t$, using (47) we have

$$
\begin{aligned}
z_{n}^{\prime}(t) & \\
= & \alpha^{\prime}(t) h_{1}(\alpha(t))\left[\left(\prod_{i=2}^{n} \mathscr{H}_{i}\right) \circ \mathscr{F}_{n(n-1)}\left[z_{n-1}\right] \mathbf{0}\right](\alpha(t)) \\
\leq & \alpha^{\prime}(t) h_{1}(\alpha(t)) \\
& \times\left[\left(\prod_{i=2}^{n} \mathscr{H}_{i}\right) \circ \mathscr{F}_{n(n-1)}\left[W_{n-1}^{-1} \circ z_{n}\right] \mathbf{0}\right](\alpha(t)),
\end{aligned}
$$

for all $t \in\left[t_{0}, T\right]$. From (49), we have

$$
\begin{gathered}
\frac{z_{n}^{\prime}(t) w_{n-1}\left(W_{1}^{-1}\left(W_{2}^{-1}\left(\cdots\left(W_{n-1}^{-1}\left(z_{n}\left(t_{n}\right)\right)\right) \cdots\right)\right)\right)}{w_{n}\left(W_{1}^{-1}\left(W_{2}^{-1}\left(\cdots\left(W_{n-1}^{-1}\left(z_{n}\left(t_{n}\right)\right)\right) \cdots\right)\right)\right)} \\
=\alpha^{\prime}(t) h_{1}(\alpha(t))\left[\left(\prod_{i=2}^{n} \mathscr{H}_{i}\right) \mathbf{1}\right](\alpha(t)),
\end{gathered}
$$

for all $t \in\left[t_{0}, T\right]$, where $\mathbf{1}$ denote the constant function $v(t) \equiv$ 1. Integrating both sides of the above inequality from $t_{0}$ to $t$, we obtain

$$
W_{n}\left(z_{n}(t)\right)-W_{n}\left(z_{n}\left(t_{0}\right)\right) \leq\left[\left(\prod_{i=1}^{n} \mathscr{H}_{i}\right) \mathbf{1}\right](\alpha(t)),
$$

for all $t \in\left[t_{0}, T\right]$. From (36), (42), (47), and (51), we have

$$
\begin{aligned}
z_{1}(t) & \leq W_{1}^{-1}\left(W_{2}^{-1}\left(\cdots\left(W_{n-1}^{-1}\left(z_{n}(t)\right)\right) \cdots\right)\right) \\
& \leq \prod_{i=1}^{n} W_{i}^{-1}\left\{W_{n}\left(z_{n}\left(t_{0}\right)\right)+\left[\left(\prod_{i=1}^{n} \mathscr{H}_{i}\right) \mathbf{1}\right](\alpha(t))\right\},
\end{aligned}
$$

for all $t \in\left[t_{0}, T\right]$. Substituting (37), (43), and (48) into (52), we have

$$
\begin{aligned}
z_{1}(t) \leq \prod_{i=1}^{n} W_{i}^{-1}\left\{W_{n}\left(\prod_{i=n-1}^{2} \mathscr{W}_{i} \circ \mathscr{W}_{1}\left(z_{1}\left(t_{0}\right)\right)(\alpha(T))\right)\right. \\
\left.+\left[\left(\prod_{i=1}^{n} \mathscr{H}_{i}\right) \mathbf{1}\right](\alpha(t))\right\},
\end{aligned}
$$

for all $t \in\left[t_{0}, T\right]$. Since $T$ is chosen arbitrarily, we have

$$
z_{1}(t) \leq \prod_{i=1}^{n} W_{i}^{-1}\left\{W_{n}\left(\prod_{i=n}^{2} \mathscr{W}_{i} \circ \mathscr{W}_{1}\left(z_{1}\left(t_{0}\right)\right)(\alpha(t))\right)\right\},
$$

for all $t \in\left[t_{0}, T\right]$. By the definition of $z_{1}$ and (32), we have

$$
\begin{aligned}
& 2 z_{1}\left(t_{0}\right)-c \\
& \quad=c+2\left\{\left(\prod_{i=1}^{n-1} \mathscr{H}_{i} \circ \mathscr{F}_{i}[u]\right) \circ \mathscr{H}_{n} \circ \mathscr{F}_{n}[u] \mathbf{0}\right\}(\alpha(T)) \\
& =z_{1}(T) .
\end{aligned}
$$

From (54) and (55), we have

$$
2 z_{1}\left(t_{0}\right)-c \leq \prod_{i=1}^{n} W_{i}^{-1}\left\{\prod_{i=n}^{2} \mathscr{W}_{i} \circ \mathscr{W}_{1}\left(z_{1}\left(t_{0}\right)\right)(\alpha(T))\right\},
$$

or

$$
\prod_{i=n}^{2} W_{i} \circ W_{1}(2 \xi-c)-\prod_{i=n}^{2} \mathscr{W}_{i} \circ \mathscr{W}_{1}(\xi)(\alpha(T)) \leq 0 .
$$

By the definition of $\mathscr{W}(\xi)$, the assumption of Theorem 3, and (57), we observe that

$$
\mathscr{W}\left(z_{1}\left(t_{0}\right)\right) \leq 0=\mathscr{W}\left(\xi_{0}\right) .
$$

By Lemma $2, \mathscr{W}(\xi)$ is increasing. From the last inequality and (31) we have the desired estimation (28).

We define the following functions:

$$
\begin{aligned}
E(u)= & W_{2}\left\{W_{1}(2 u-c)\right\} \\
& -W_{2}\left\{W_{1}(u)+\left(\mathscr{H}_{1} f_{1}(t)\right)(\alpha(T))\right\} \\
& -\mathscr{H}_{1} \circ\left(\mathscr{H}_{2} \mathbf{1}\right)(\alpha(T)),
\end{aligned}
$$

for all $u>c$, where $W_{i}, i=1,2$ are defined by (13) and (14), respectively. 
Example 5. Let $n=2, f_{1}(t), h_{i}(t), \varphi_{i}, W_{i}, i=1,2, \alpha$ be as in Theorem $3 ; c$ is a positive constant. Suppose that the function $E(u)$ is increasing and $E(u)=0$ has a solution $c$ for $u>c$. If $u(t)$ satisfies $(22)$, then

$$
\begin{aligned}
& u(t) \\
& \leq W_{1}^{-1}\left\{W _ { 2 } ^ { - 1 } \left\{W_{2}\left\{W_{1}(c)+\left(\mathscr{H}_{1} f_{1}(t)\right)(\alpha(t))\right\}\right.\right. \\
& \left.\left.-\mathscr{H}_{1} \circ\left(\mathscr{H}_{2} \mathbf{1}\right)(\alpha(t))\right\}\right\}, \quad \forall t \in\left[t_{0}, T\right],
\end{aligned}
$$

where $W_{i}^{-1}(i=1,2)$ are inverse functions of $W_{i}$, respectively.

Remark 6. If $w_{1}=w_{2}$ in Example 5, then the result in Example 5 will yield the conclusion that appeared in Theorem $2.1^{\prime}$ in [17]. Since if $w_{1}=w_{2}$, then $W_{2}(u)=u-u_{2}, W_{2}^{-1}(u)=$ $u+u_{2}$, from (60), we have $u(t) \leq W_{1}^{-1}\left\{W_{1}(c)+H_{1}(t)+H_{2}(t)\right\}$ for all $t \in\left[t_{0}, T\right]$.

\section{Application}

In this section, we apply our result in Theorem 3 to investigate the retarded Volterra-Fredholm integral equations

$$
\begin{aligned}
& x(t)=x_{0} \\
& +\int_{t_{0}}^{t} F_{1}\left\{s, x(s-d(s)), \int_{t_{0}}^{s} F_{2}[\tau, x(\tau-d(\tau))] d \tau\right\} d s \\
& \quad+\int_{t_{0}}^{T} F_{1}\left\{s, x(s-d(s)), \int_{t_{0}}^{s} F_{2}[\tau, x(\tau-d(\tau))] d \tau\right\} d s,
\end{aligned}
$$

for $t \in\left[t_{0}, T\right]$, where $x \in C(I, \mathbf{R}), d \in C^{1}(I, I)$ is nondecreasing with $t-d(t) \geq t_{0}, d\left(t_{0}\right)=0, d^{\prime}(t)<1, F_{1} \in$ $C\left(I \times \mathbf{R}^{2}, \mathbf{R}\right)$, and $F_{2} \in C(I \times \mathbf{R}, \mathbf{R})$. Let $\beta(t)=t-d(t)$; then $\beta(t) \in C^{1}(I, I), \beta(t) \leq t$. Since $\beta^{\prime}(t)=1-d^{\prime}(t)>0, \beta(t)$ is an increasing and invertible function.

The following corollary gives the bound on the solution of (61).

Corollary 7. Suppose that the $F_{1}, F_{2}$ in (61) satisfy the conditions

$$
\begin{gathered}
\left|F_{1}(s, x, y)\right| \leq h_{1}(s)\left[f_{1}(s) w_{1}(|x|)+|y|\right], \\
\left|F_{2}(s, x)\right| \leq h_{2}(s) w_{2}(|x|),
\end{gathered}
$$

where $f_{1}(s), h_{1}(s), h_{2}(s), w_{1}(s)$, and $w_{2}(s)$ are as in Theorem 3; let $M=\max _{t \in\left[t_{0}, T\right]}\left(1 / \beta^{\prime}\left(\beta^{-1}(t)\right)\right)<\infty$. Assume that the function

$$
\begin{aligned}
E^{*}(u)= & W_{2}\left[W_{1}\left(2 u-\left|x_{0}\right|\right)\right] \\
& -W_{2}\left[W_{1}(u)+\int_{\beta\left(t_{0}\right)}^{\beta(T)} M h_{1}(s) f_{1}(s) d s\right] \\
& -\int_{\beta\left(t_{0}\right)}^{\beta(T)} M h_{1}(s) \times\left[\int_{\beta\left(t_{0}\right)}^{s} M h_{2}(\tau) d \tau\right] d s
\end{aligned}
$$

is increasing and $E^{*}(u)=0$ has a solution c for $u>\xi_{0}$. If $x(t)$ is a solution of (61), then

$$
\begin{aligned}
& \qquad x(t) \mid \leq W_{1}^{-1} \\
& \times\left\{W _ { 2 } ^ { - 1 } \left[W _ { 2 } \left[W_{1}(c)\right.\right.\right. \\
& \left.\quad+\int_{\beta\left(t_{0}\right)}^{\beta(t)} M h_{1}\left(\beta^{-1}(s)\right) f_{1}\left(\beta^{-1}(s)\right) d s\right] \\
& +\int_{\beta\left(t_{0}\right)}^{\beta(t)} M h_{1}\left(\beta^{-1}(s)\right) \\
& \left.\left.\quad \times\left[\int_{\beta\left(t_{0}\right)}^{s} M h_{2}\left(\beta^{-1}(\tau)\right) d \tau\right] d s\right]\right\} \\
&
\end{aligned}
$$

where $W_{1}, W_{2}, W_{1}^{-1}$, and $W_{2}^{-1}$ are as in Theorem 3 .

Proof. Using the conditions (62)-(63) we have

$$
\begin{aligned}
& |x(t)| \leq\left|x_{0}\right| \\
& +\int_{t_{0}}^{t} h_{1}(s)\left[f_{1}(s) w_{1}(|x(s-d(s))|)\right. \\
& \left.+\int_{t_{0}}^{s} h_{2}(\tau) w_{2}(|x(\tau-d(\tau))|) d \tau\right] d s \\
& +\int_{t_{0}}^{T} h_{1}(s)\left[f_{1}(s) w_{1}(|x(s-d(s))|)\right. \\
& \left.+\int_{t_{0}}^{s} h_{2}(\tau) w_{2}(|x(\tau-d(\tau))|) d \tau\right] d s \\
& =\left|x_{0}\right|+\int_{t_{0}}^{t} h_{1}(s)\left[f_{1}(s) w_{1}(|x(\beta(s))|)\right. \\
& \left.+\int_{t_{0}}^{s} h_{2}(\tau) w_{2}(|x(\beta(\tau))|) d \tau\right] d s \\
& +\int_{t_{0}}^{T} h_{1}(s)\left[f_{1}(s) w_{1}(|x(\beta(s))|)\right. \\
& \left.+\int_{t_{0}}^{s} h_{2}(\tau) w_{2}(|x(\beta(\tau))|) d \tau\right] d s \\
& \leq\left|x_{0}\right| \\
& +\int_{t_{0}}^{t} h_{1}(s)\left[f_{1}(s) w_{1}(|x(\beta(s))|)\right. \\
& \left.+\int_{\beta\left(t_{0}\right)}^{\beta(s)} M h_{2}\left(\beta^{-1}(\tau)\right) w_{2}(|x(\tau)|) d \tau\right] d s
\end{aligned}
$$




$$
\begin{aligned}
& +\int_{t_{0}}^{T} h_{1}(s)\left[f_{1}(s) w_{1}(|x(\beta(s))|)\right. \\
& \left.\quad+\int_{\beta\left(t_{0}\right)}^{\beta(s)} M h_{2}\left(\beta^{-1}(\tau)\right) w_{2}(|x(\tau)|) d \tau\right] d s \\
& \leq\left|x_{0}\right|+\int_{\beta\left(t_{0}\right)}^{\beta(t)} M h_{1}\left(\beta^{-1}(s)\right) \\
& \times\left[f_{1}\left(\beta^{-1}(s)\right) w_{1}(|x(s)|)\right. \\
& \left.+\int_{\beta\left(t_{0}\right)}^{s} M h_{2}\left(\beta^{-1}(\tau)\right) w_{2}(|x(\tau)|) d \tau\right] d s \\
& +\int_{\beta\left(t_{0}\right)}^{\beta(T)} M h_{1}\left(\beta^{-1}(s)\right) \\
& \quad \times\left[f_{1}\left(\beta^{-1}(s)\right) w_{1}(|x(s)|)\right. \\
& \left.\quad+\int_{\beta\left(t_{0}\right)}^{s} M h_{2}\left(\beta^{-1}(\tau)\right) w_{2}(|x(\tau)|) d \tau\right] d s
\end{aligned}
$$

for $t \in\left[t_{0}, T\right]$, where several changes of variables are made. Applying the result of Theorem 3 to the last inequality, we obtain the desired estimation (65).

\section{Conflict of Interests}

The authors declare that there is no conflict of interests regarding the publication of this paper.

\section{Acknowledgments}

The authors are very grateful to the editor and the referees for their helpful comments and valuable suggestions. This research was supported by National Natural Science Foundation of China (Project no. 11161018) and the NSF of Guangxi Zhuang Autonomous Region (no. 2012GXNSFAA053009).

\section{References}

[1] T. H. Gronwall, "Note on the derivatives with respect to a parameter of the solutions of a system of differential equations," Annals of Mathematics, vol. 20, no. 4, pp. 292-296, 1919.

[2] R. Bellman, "The stability of solutions of linear differential equations," Duke Mathematical Journal, vol. 10, pp. 643-647, 1943.

[3] I. Bihari, "A generalization of a lemma of Bellman and its application to uniqueness problems of differential equations," Acta Mathematica Academiae Scientiarum Hungaricae, vol. 7, pp. 8194, 1956.

[4] M. Pinto, "Integral inequalities of Bihari-type and applications," Funkcialaj Ekvacioj, vol. 33, no. 3, pp. 387-403, 1990.

[5] O. Lipovan, "A retarded Gronwall-like inequality and its applications," Journal of Mathematical Analysis and Applications, vol. 252, no. 1, pp. 389-401, 2000.
[6] R. P. Agarwal, S. Deng, and W. Zhang, "Generalization of a retarded Gronwall-like inequality and its applications," Applied Mathematics and Computation, vol. 165, no. 3, pp. 599-612, 2005.

[7] W. N. Zhang, "Projected Gronwall's inequality," Journal of Mathematical Research and Exposition, vol. 17, no. 2, pp. 257-260, 1997 (Chinese).

[8] W. Zhang and S. Deng, "Projected Gronwall-Bellman's inequality for integrable functions," Mathematical and Computer Modelling, vol. 34, no. 3-4, pp. 393-402, 2001.

[9] L. Zhou, K. Lu, and W. Zhang, "Roughness of tempered exponential dichotomies for infinite-dimensional random difference equations," Journal of Differential Equations, vol. 254, no. 9, pp. 4024-4046, 2013.

[10] Q. H. Ma and E. H. Yang, "Estimates on solutions of some weakly singular Volterra integral inequalities," Acta Mathematicae Applicatae Sinica, vol. 25, no. 3, pp. 505-515, 2002.

[11] Q.-H. Ma and J. Pečarić, "Some new explicit bounds for weakly singular integral inequalities with applications to fractional differential and integral equations," Journal of Mathematical Analysis and Applications, vol. 341, no. 2, pp. 894-905, 2008.

[12] J. Henderson and S. Hristova, "Nonlinear integral inequalities involving maxima of unknown scalar functions," Mathematical and Computer Modelling, vol. 53, no. 5-6, pp. 871-882, 2011.

[13] Y. Yan, "Nonlinear Gronwall-Bellman type integral inequalities with maxima," Mathematical Inequalities \& Applications, vol. 16, no. 3, pp. 911-928, 2013.

[14] D. Bainov and P. Simeonov, Integral Inequalities and Applications, vol. 57 of Mathematics and Its Applications, Kluwer Academic, Dordrecht, The Netherlands, 1992.

[15] B. G. Pachpatte, Inequalities for Differential and Integral Equations, Academic Press, London, UK, 1998.

[16] B. G. Pachpatte, "Explicit bound on a retarded integral inequality," Mathematical Inequalities \& Applications, vol. 7, no. 1, pp. 7-11, 2004.

[17] Q. H. Ma and J. Pečarić, "Estimates on solutions of some new nonlinear retarded Volterra-Fredholm type integral inequalities," Nonlinear Analysis: Theory, Methods \& Applications, vol. 69, no. 2, pp. 393-407, 2008.

[18] A. Abdeldaim and M. Yakout, "On some new integral inequalities of Gronwall-Bellman-Pachpatte type," Applied Mathematics and Computation, vol. 217, no. 20, pp. 7887-7899, 2011.

[19] W. Wang, D. Huang, and X. Li, "Generalized retarded nonlinear integral inequalities involving iterated integrals and an application," Journal of Inequalities and Applications, vol. 2013, article 376, 2013. 


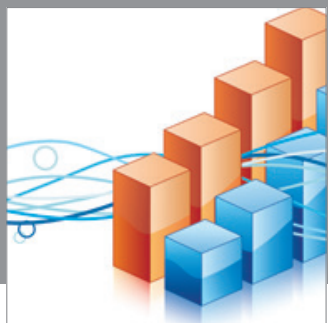

Advances in

Operations Research

mansans

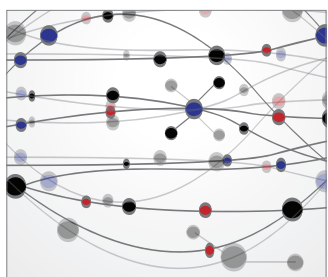

The Scientific World Journal
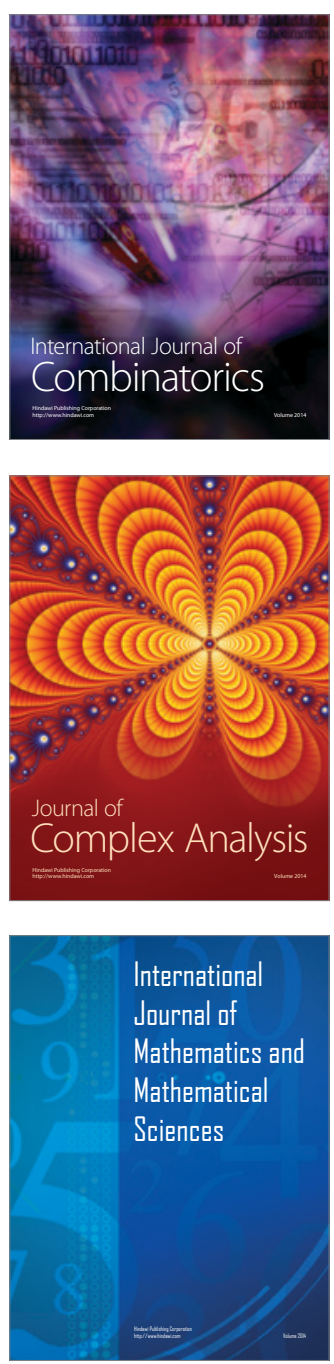
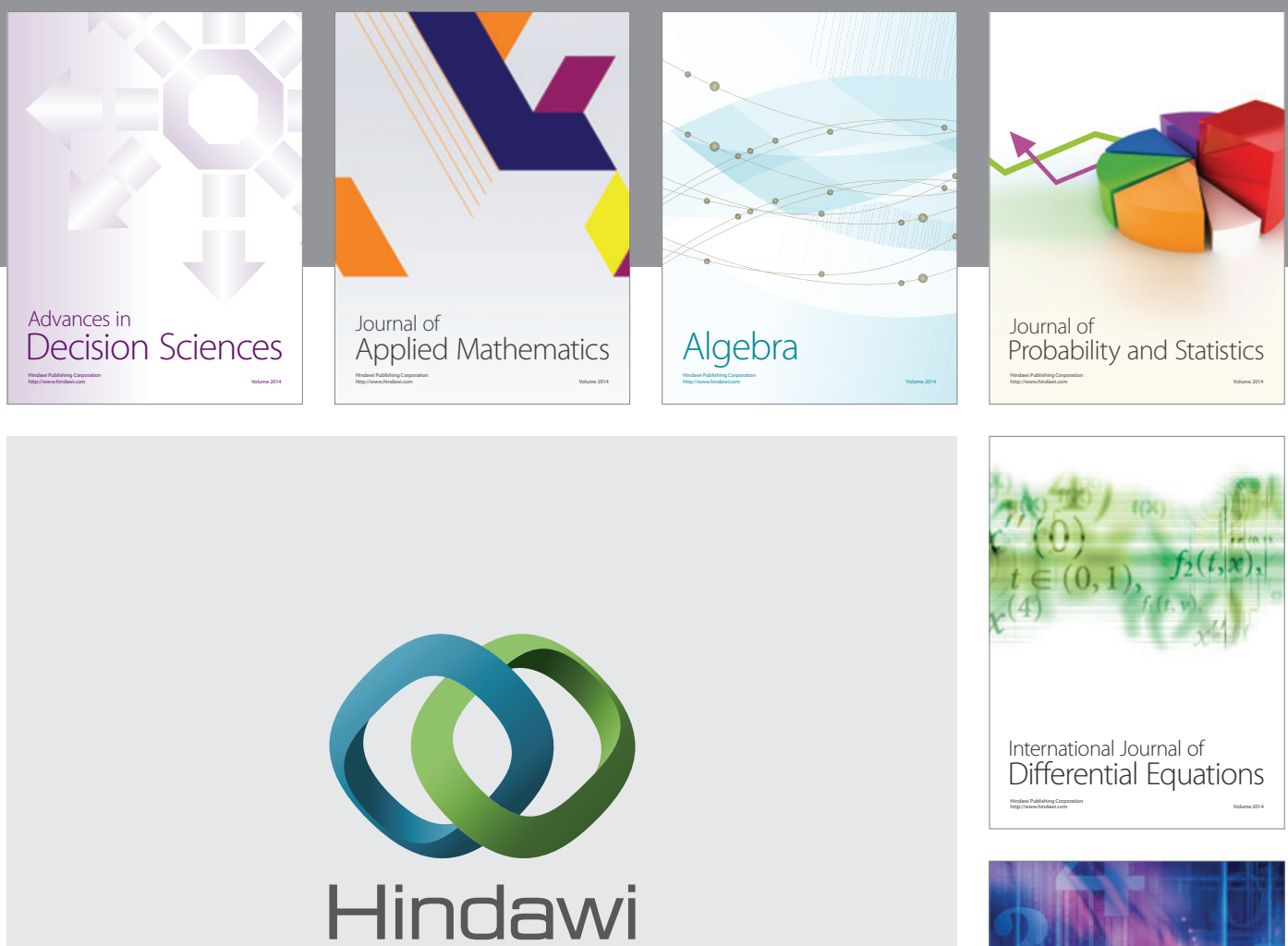

Submit your manuscripts at http://www.hindawi.com
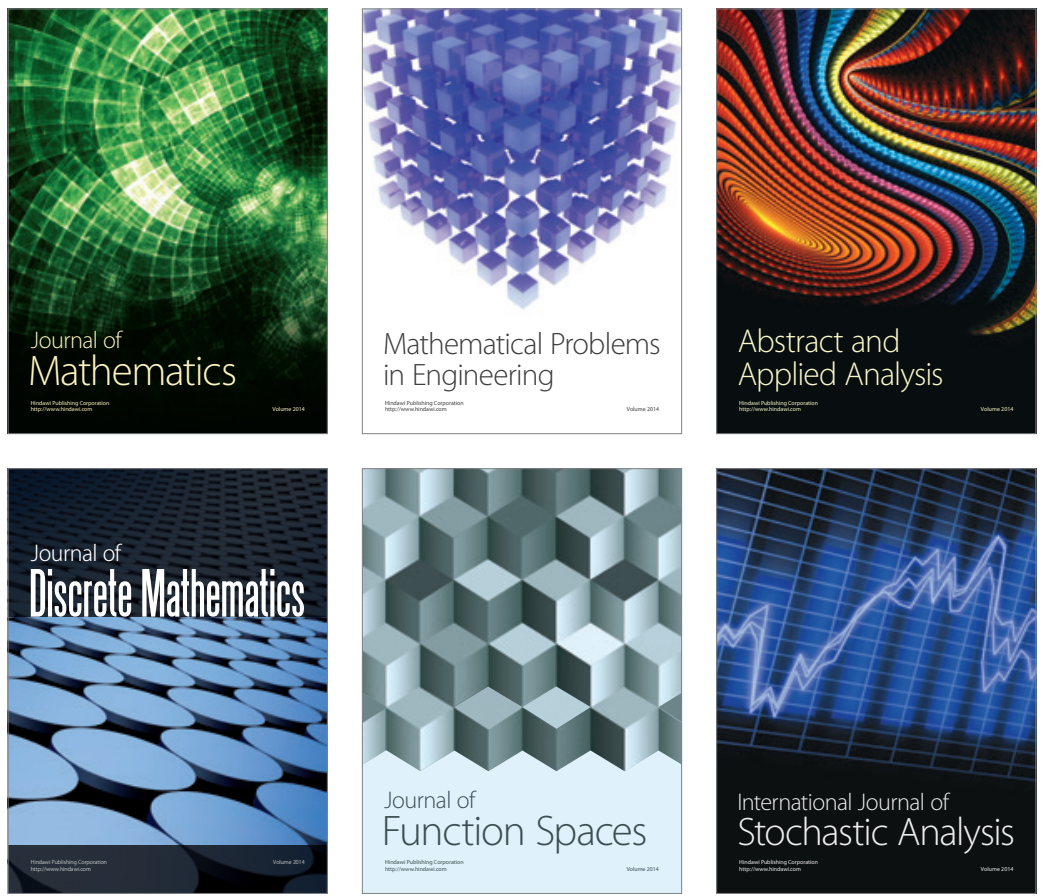

Journal of

Function Spaces

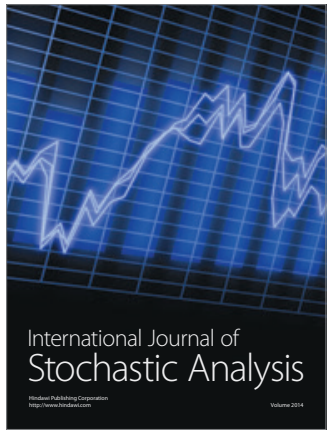

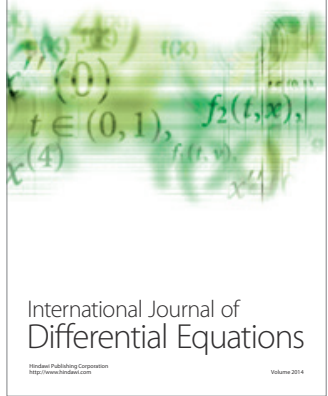
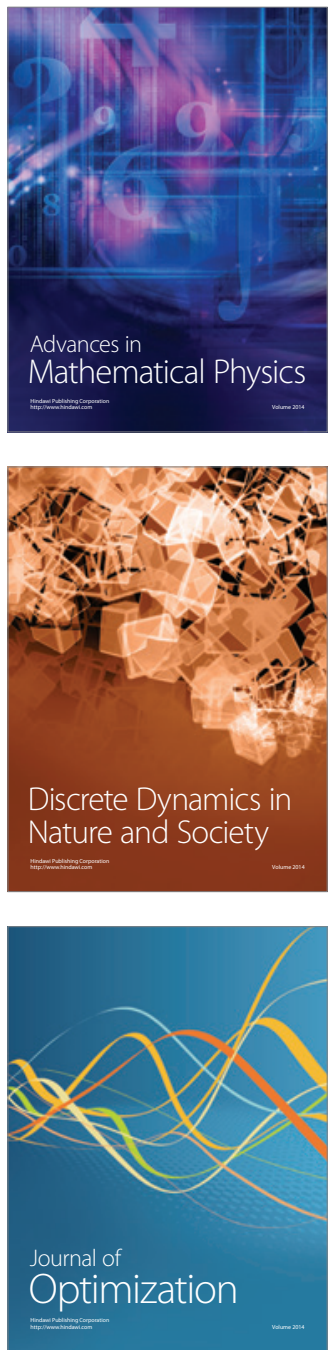